\title{
Supporting Information \\ Surface Grafted Antimicrobial Polymer \\ Networks with High Abrasion Resistance
}

Jing Gao,${ }^{\dagger}$ N. Eric Huddleston, ${ }^{\S}$ Evan M. White, ${ }^{\dagger}$ Jitendra Pant,${ }^{\ddagger}$

Hitesh Handa, ${ }^{\ddagger}$ Jason Locklin $*, \dagger$,

${ }^{\dagger}$ Department of Chemistry, University of Georgia, Athens, Georgia, 30602, United States

${ }^{\sharp}$ School of Biological and Biochemical Engineering, College of Engineering, University

of Georgia, Athens, Georgia, 30602, United States

$\S$ Department of Chemistry and Biochemistry, University of North Georgia, Dahlonega,

Georgia, 30597 


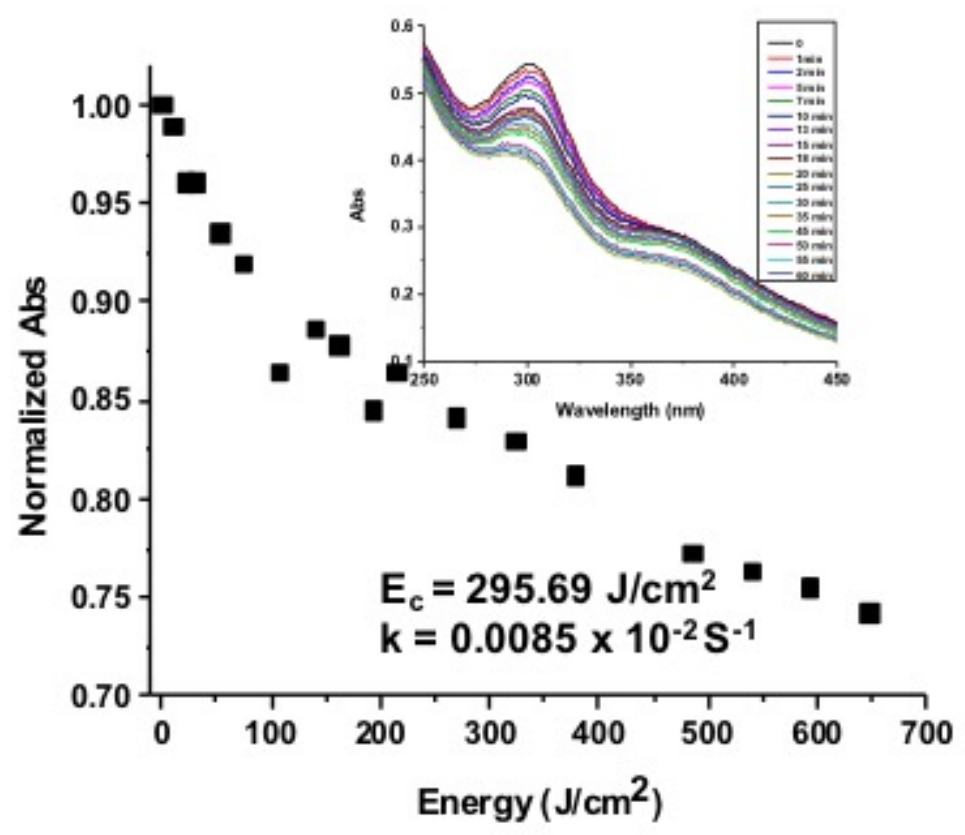

Figure S1. BP conversion of AmBP monitored via UV-Vis spectrometry of $n-\pi^{*}$ peak at $303 \mathrm{~nm}$ (Inset: UV-Vis Spectra of AmBP).

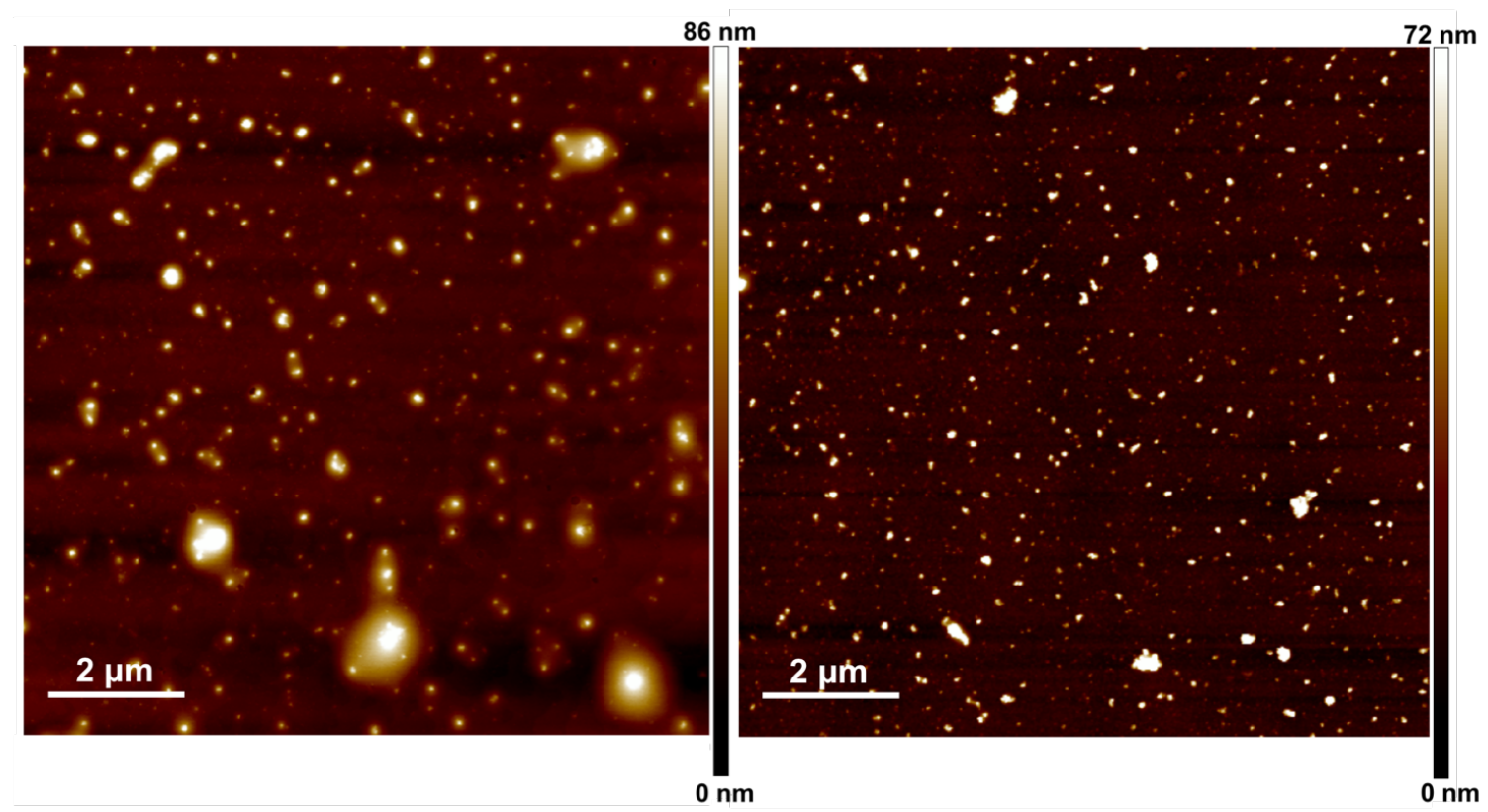

Figure S2. AFM height image (scan size $=10 \mu \mathrm{m}$ ) for the film of BPAM (A) before irradiation (RMS roughness $9.78 \mathrm{~nm}$ ) and (B) after irradiation (RMS roughness $8.15 \mathrm{~nm}$ ). 


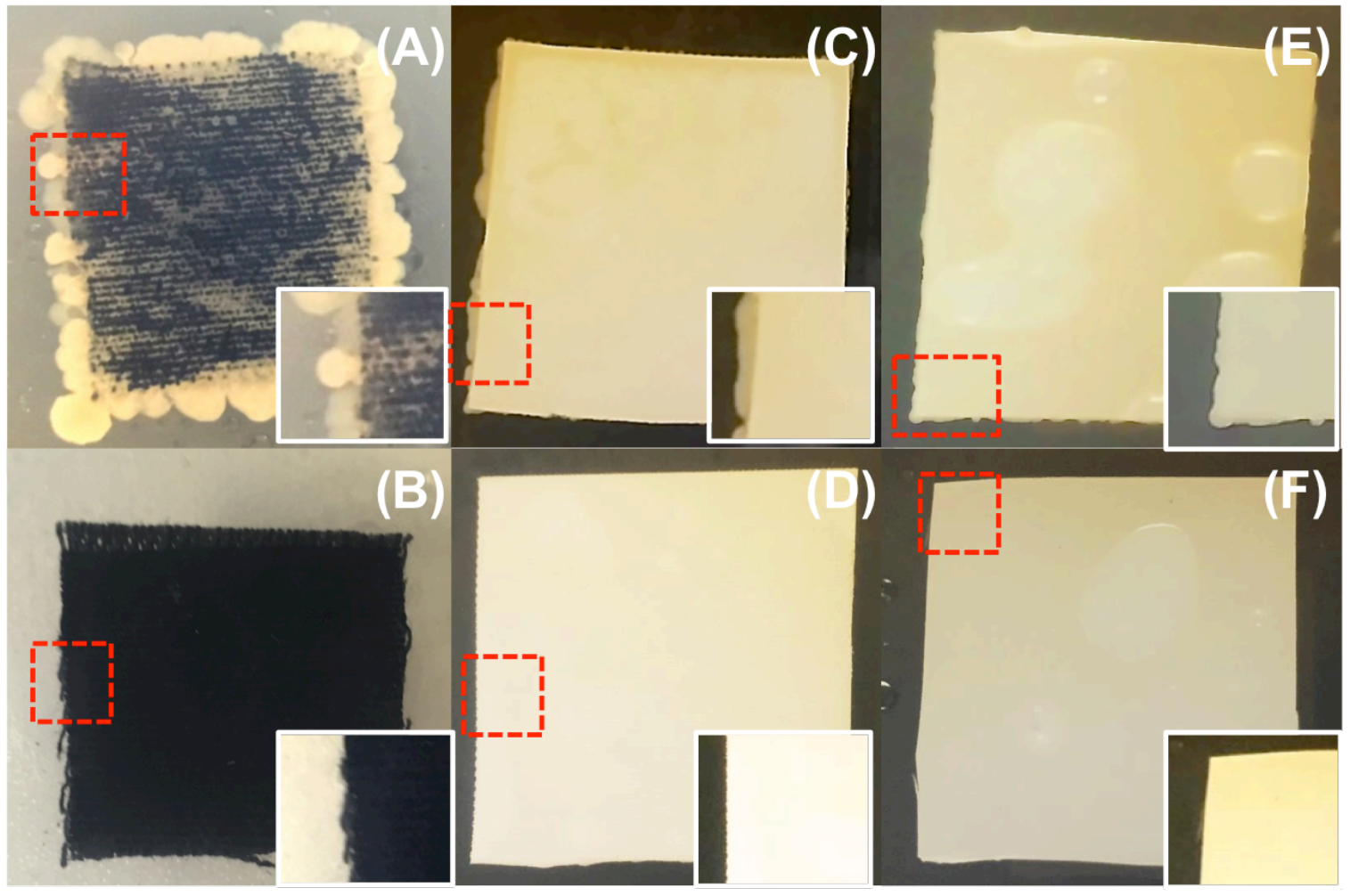

Figure S3. Digital pictures of the plastic and fabric substrates sprayed with E. coli suspension and incubated overnight at $37^{\circ} \mathrm{C}$ : (A) uncoated cotton, (B) BPAM coated cotton, (C) uncoated PVA, (D) BPAM coated PVA, (E) uncoated PE, (F) BPAM coated PE. Insets: zoom-in images of the highlighted squared areas. 


\section{(A)}

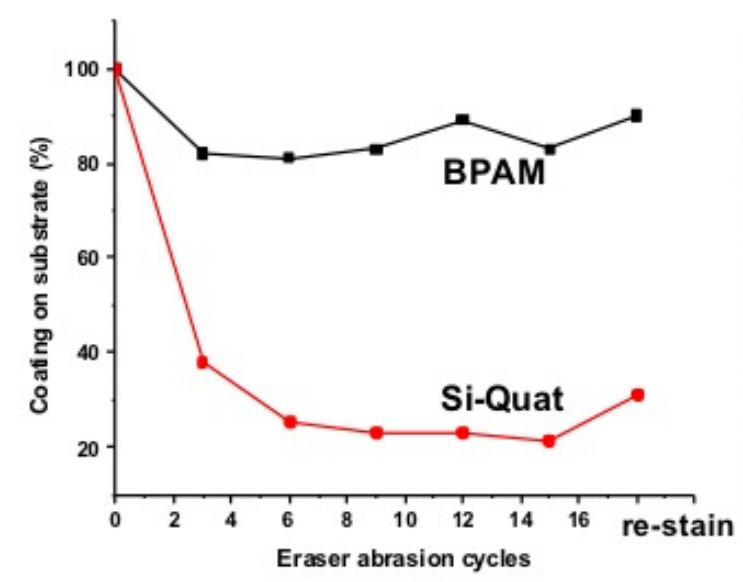

(B)

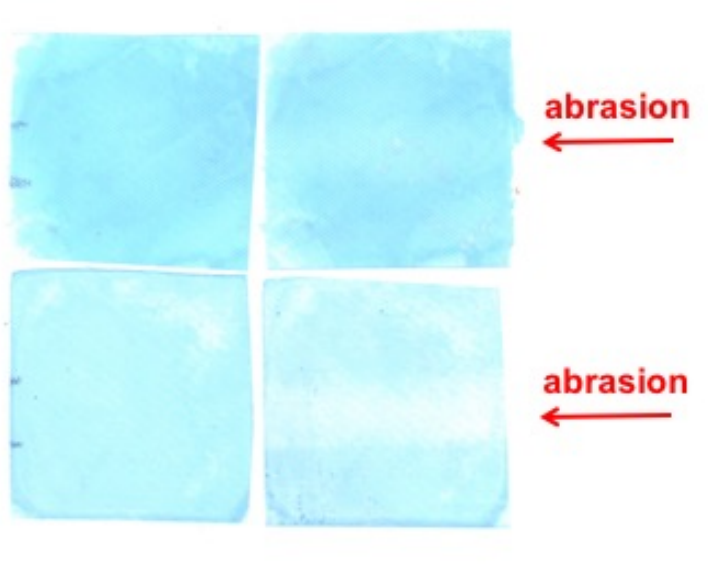

Figure S4. Plots of percentage of remaining BPAM ( ) and Si-Quat (O) on PVA over mechanical abrasion cycles (A) and optical images of the samples before and after 15 abrasion cycles. 